\section{The Home is the New Cancer Center}

\author{
Nathan R. Handley, MD, MBA; Justin E. Bekelman, MD; and Adam F. Binder, MD
}

A s oncologists in the era of coronavirus disease 2019 (COVID-19), we are tasked with caring for patients who are at high risk for adverse effects from the novel coronavirus (SARS-CoV-2). ${ }^{1}$ Patients with cancer face fear and anxiety related to their immunocompromised status, and early data suggest patients with cancer may be more susceptible to infection with SARS-CoV-2. ${ }^{2}$ During this pandemic, a new vuInerability has arisen: how can patients receive life-sustaining treatment for cancer if they cannot get to the clinic or if the system is overwhelmed by patients with COVID-19? How can patients stay in what may be the safest place for them-the home-while still receiving high-quality care?

COVID-19 provides an opportunity to recognize and improve on shortcomings in our cancer care delivery system: interacting with the health system puts already tenuous patients at risk for nosocomial infection, high-quality cancer care requires a high level of coordination that is challenging to achieve, and accessing care can be difficult or impossible for some individuals. Moving components of cancer care into the home can facilitate high-touch care, minimize risk by reducing unnecessary exposures to the health system, and improve access to treatment. Cancer care at home can augment or replace current models for clinic visits, chemotherapy, and acute care, deleveraging healthcare facilities as primary sites of care while ensuring safety for patients and providers, and improving patient-centeredness of care. Although important during a pandemic, cancer care at home can also have a lasting impact when healthcare returns to "normal operations." This commentary describes home-based approaches for clinic visits, chemotherapy, and acute care; examines concerns stakeholders have raised about implementation of these approaches; and evaluates needs for the sustainability of cancer care at home after resolution of the pandemic.

\section{Clinic Visits}

The transition from in-person clinic visits to telemedicine-based virtual visits has been rapid and dramatic since the pandemic began. At Jefferson Health, JeffConnect, a telemedicine platform for virtual visits, has seen a 20 -fold increase in use. This modality is appropriate for patients presenting for routine follow-up care or receiving oral chemotherapy, and can be readily adapted for patients receiving infusional treatments. Similarly, we have found that supportive care services-including peer support groups, patient-facing educational courses, and palliative care appointments-readily lend themselves to the virtual format.

Hollander and Sites ${ }^{3}$ recently outlined 5 concerns regarding telemedicine: it's too difficult, it's too transactional, the physical examination is inadequate, it's ineffective, and it's not financially viable. As the uptake of telehealth during the pandemic has soared, clinicians and patients are now recognizing the many positive attributes of telehealth and its clinical effectiveness. Although some of the momentum telemedicine has experienced during COVID-19 is due to temporary waivers that facilitate reimbursement, telemedicine has now been thrust into the mainstream, and the gains will inevitably pave the way for broader uptake in the future.

\section{Chemotherapy}

Patients are encouraged to shelter in place to minimize exposures to SARS-CoV-2. For patients who require infusional chemotherapy, however, some interaction with the health system is inevitable. Yet receiving treatment within a hospital or an infusion

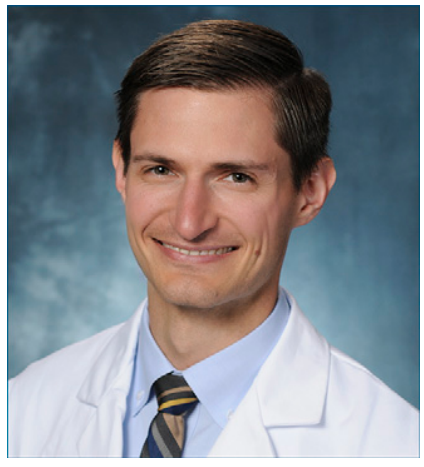

NATHAN R. HANDLEY, MD, MBA

Nathan R. Handley, MD, MBA, is Assistant Professor in the Department of Medical Oncology at Thomas Jefferson University. $\mathrm{He}$ is a board certified oncologist specializing in genitourinary malignancies. His research interests involve the development and implementation of strategies to improve cancer care delivery.

doi: $10.6004 /$ jnccn.2020.7626

The ideas and viewpoints expressed in this commentary are those of the author and do not necessarily represent any policy, position, or program of NCCN. 


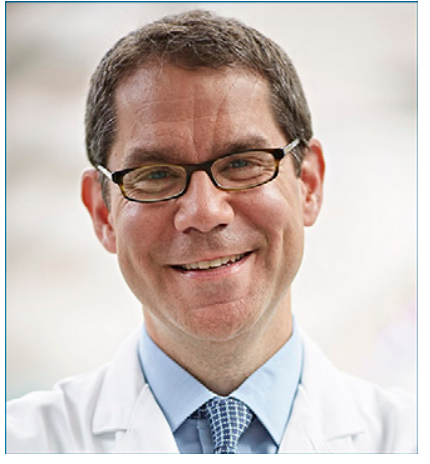

JUSTIN E. BEKELMAN, MD

Justin E. Bekelman, MD, is Director of the Penn Center for Cancer Care Innovation at the Abramson Cancer Center, Professor in the Departments of Radiation Oncology and Medical Ethics and Health Policy at the Perelman School of Medicine, Faculty in the Center for Health Incentives and Behavioral Economics, and Senior Fellow at the Penn Center for Precision Medicine and the Leonard Davis Institute for Health Economics, all at the University of Pennsylvania. Dr. Bekelman leads research programs to improve the length and quality of life for patients with cancer. His research focuses on healthcare delivery, payment reform, and cancer care transformation, integrating methods from the fields of innovation, epidemiology, clinical trials, behavioral and health economics, and public policy. center carries nosocomial risk: in the infusion suite, dozens of potentially immunocompromised patients are collocated with a variety of healthcare providers who may be vectors for SARS-CoV-2. For these patients, home-based chemotherapy, administered by a trained team, can be considered. Chemotherapy at home remains uncommon in the United States, although models for its use exist globally. ${ }^{4}$ In these models, home-based chemotherapy has been safe, was preferred by patients, and could be administered at lower cost. The Christie NHS Foundation Trust, the largest single-site cancer center in Europe, recently described "Christie at Home," a model of home-based anticancer therapy designed to reduce pressure on clinics and improve the patient experience. ${ }^{5}$ In this model, a trained nurse can deliver 7 subcutaneous or intramuscular treatments or 4 or 5 intravenous treatments per day, with real-time documentation completed via 4G-enabled laptops. Christie at Home delivered 11,906 in-home treatments between 2015 and 2019; in 2018, 100\% of surveyed patients receiving care at home rated their care as excellent and indicated a preference for home-based care over other sites. In some cases, a physical care team may not be required for administration of therapy, which may further streamline treatment. For instance, at Jefferson Health, our specialty pharmacy can deliver certain injectable agents, such as leuprolide, to the patient's home for selfadministration.

Concerns surround home-based chemotherapy as well. These concerns include fear that it is not safe, that logistics are insurmountable, that it cannot be reimbursed, and that patients do not want it. Successful models, both domestically and abroad, address these concerns. For example, Penn Medicine is now scaling a home-infusion demonstration program called "Cancer Care at Home" (CC@H). This program provides 13 different cancer drugs, which are typically provided in outpatient cancer settings, to patients with lymphoma or breast, prostate, lung, or head and neck cancer for home administration. Patients discuss $\mathrm{CC} @ \mathrm{H}$ with their provider, who then prescribes home treatment in the electronic medical record. After insurance authorization and discussion of out-of-pocket costs by Penn Home Infusion Therapy, a courier delivers the treatment to the patient's home (up to 2 days in advance); the treatment is then administered in the home by a nurse. ${ }^{6}$

\section{Acute Care}

In the era of COVID-19, minimizing unplanned and potentially preventable acute care use for patients with cancer is particularly important. These encounters, including emergency department (ED) visits, acute hospitalizations, and 30-day rehospitalizations, use limited resources and put patients, staff, and providers at increased risk for infection. However, the desire to prevent unnecessary acute care use by patients with cancer is not unique to the pandemic.

Many of these patients can be managed in the home setting via hospital-athome models. ${ }^{4}$ Although these programs are currently uncommon in the United States, they do exist and are increasing in number. For example, Huntsman Cancer Institute currently operates Huntsman at Home $(\mathrm{H} @ \mathrm{H})$, which provides acute care services for oncology patients who live within 20 miles of the institute. This program was born out of a need to address full bed occupancy and after-hours ED visits. Both of these problems are highly pertinent during a pandemic. $\mathrm{H} @ \mathrm{H}$ reports reduced hospitalizations, ED visits, and total costs among patients who participate in the program. ${ }^{7}$

Concerns about safety, feasibility, patient-centeredness, and reimbursement exist for cancer hospital-at-home programs as well. Despite these concerns, however, hospital-at-home models exist and are expanding. Reimbursement may be the biggest barrier, but payment models are developing. For example, in 2017, the Physician-Focused Payment Model Technical Advisory Committee recommended a bundled payment for home-based acute care (consisting of acute hospital-level care 
and 30 days of transition services) to be implemented as an alternative payment model. ${ }^{8}$ Similar payment models exist in collaborations between health systems and private payers. $^{9}$

Cancer care at home is not a replacement for all care. Much of cancer care should not transition to the home, and identifying which treatments, and for which patients, can be safely and effectively delivered at home is crucial. Cancer care at home may not be appropriate for every eligible patient and will not be appropriate for every treatment. Shared decision-making between clinicians, patients, and family members can help tailor care to the needs of the individual patient. Ultimately, however, reserving facilities for those who truly need facility-level care will best serve all stakeholders.

\section{Conclusions}

COVID-19 has illuminated the need for reform of the cancer care delivery system. By moving components of care to the home, the current system could effectively engage patients and improve access to care while reducing unnecessary and risky exposure to the healthcare system. Furthermore, increased time at home would align with a new proposed patient-determined quality indicator for cancer care: days at home in the last 6 months of life. ${ }^{10}$

Challenges remain if cancer care at home, which has the potential to be of critical importance during the pandemic, is to become a standard option for cancer care delivery after the pandemic. To be sustainable, virtual visits, chemotherapy, and acute care at home require reallocation of resources, development of infrastructure, and durable payment reform. COVID-19 can be a catalyst for such change. We should leverage this momentum to transform care for our patients.

Disclosures: Dr. Bekelman has disclosed that he has received honoraria from Optum and CVS Health Dr. Bekelman and Dr. Binder have disclosed that they have no financial interests, arrangement affiliations, or commercial interests with the manufacturers of any products discussed in this article or their competitors.

Correspondence: Nathan R. Handley, MD, MBA, Department of Medical Oncology, Thomas Jefferson University, 1025 Walnut Street, Suite 700, Philadelphia, PA 19107. Email: Nathan.Handley@jefferson.edu

\section{References}

1. Wang D, Hu B, Hu C, et al. Clinical characteristics of 138 hospitalized patients with 2019 novel coronavirus-infected pneumonia in Wuhan, China. JAMA 2020;323:1061-1069

2. $\mathrm{Yu}$ J, Ouyang $W$, Chua MLK, et al. SARS-CoV-2 transmission in patients with cancer at a tertiary care hospital in Wuhan, China. JAMA Oncol 2020;6:1108.

3. Hollander JE, Sites FD. The Transition from Reimagining to Recreating Health Care Is Now. Accessed June 30, 2020. Available at: https://catalyst.nejm.org/doi/full/10.1056/CAT.20.0093

4. Handley NR, Bekelman JE. The oncology hospital at home. J Clin Oncol 2019;37:448-452.

5. Burns V, Misra V, Paton N. Systemic anti-cancer therapy delivery in the home: a service model. Br J Nurs 2020;29:S22-29.

6. Laughlin Al, Begley M, Delaney T, et al. Accelerating the Delivery of Cancer Care at Home During the Covid-19 Pandemic. Accessed July 15, 2020. Available at: https://catalyst.nejm.org/doi/full/10.1056/ CAT.20.0258

7. Mooney K, Titchener K, Haaland B, et al. The oncology hospital at home: health care utilization outcomes from the Huntsman at home trial [published online May 25, 2020]. J Clin Oncol, doi: 10.1200/ JCO.2020.38.15_suppl.7000 https://ascopubs.org/doi/abs/10.1200/JCO.2020.38.15_suppl.7000

8. Physican-Focused Payment Model Technical Advisory Committee. Report to the Secretary of Health and Human Services. Accessed July 15, 2020. Available at: https://aspe.hhs.gov/system/files/pdf/ 255906/MtSinaiHAHReportSecretary.pdf

9. Murali N, Messina T. No place like home: bringing inpatient care to the patient [published online August 15, 2019]. NJEM Catalyst, doi: 10.1056/CAT.19.0628 10.1056/CAT.19.0628

10. Andersen SK, Croxford R, Earle CC, et al. Days at home in the last 6 months of life: a patient-determined quality indicator for cancer care. J Oncol Pract 2019;15:e308-315.

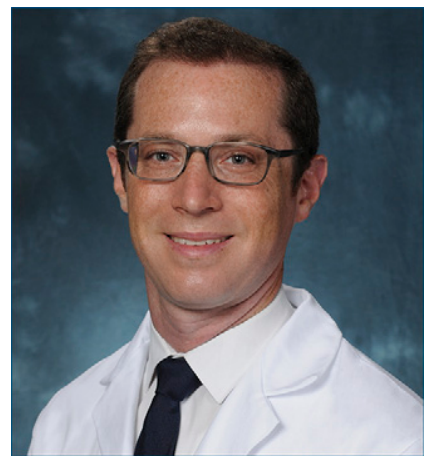

ADAM F. BINDER, MD

Adam F. Binder, MD, is Assistant Professor in the Department of Medical Oncology and Quality Officer in the Division of Hematologic Malignancies at Thomas Jefferson University. He is a board certified hematologist specializing in multiple myeloma. His interests include quality improvement and cancer care delivery research. 